$\Lambda 4$

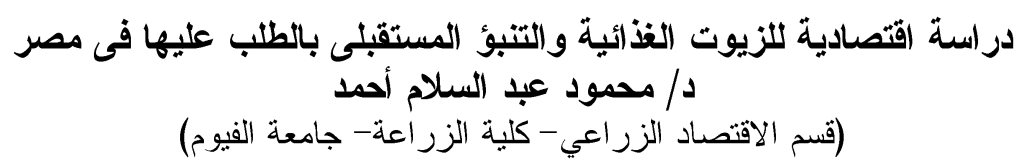

يُعتبر قطاع الإنتاج النباتي ركيزة أساسية للإنتاج الزراعي حيث بلغت قيمته حوالي الزئل

مقدمة:

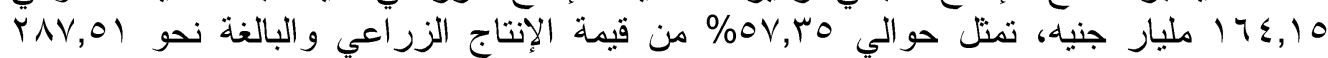

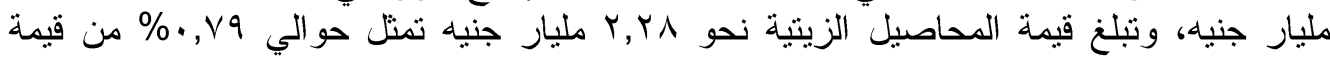

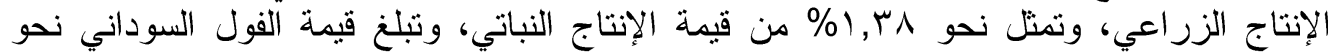

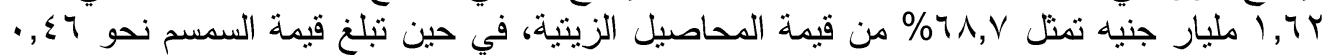

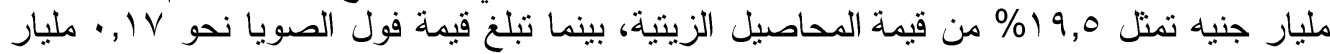

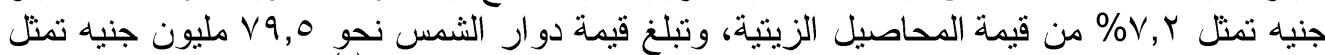

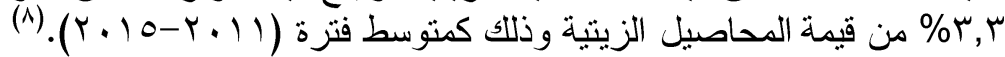

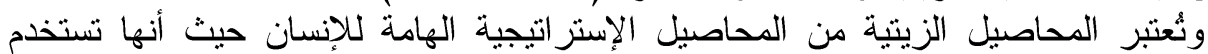

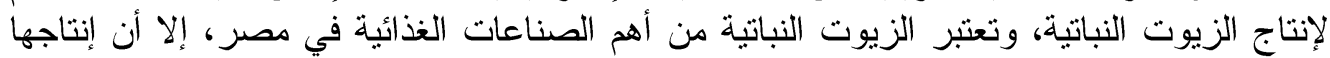

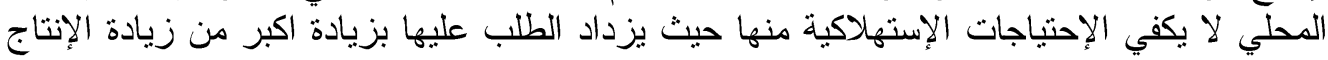

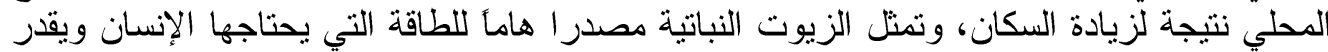

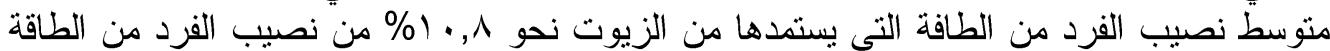

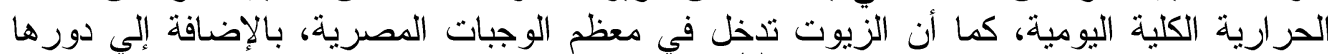

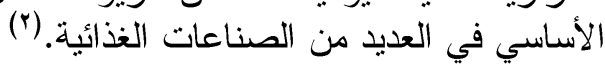

وعلي الرغم من تعدُد المحاصيل الزيتية التي يمكن زر اعتها إلا أن مساحة تلك التهائ المحاصيل

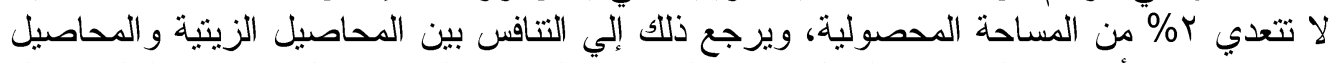

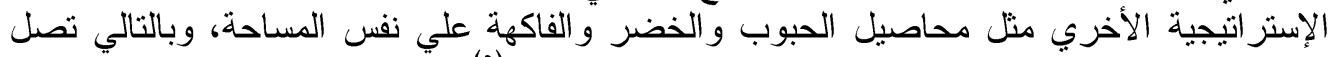

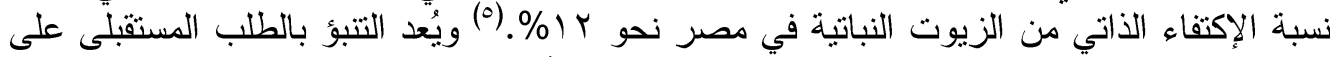

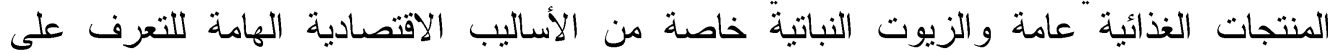

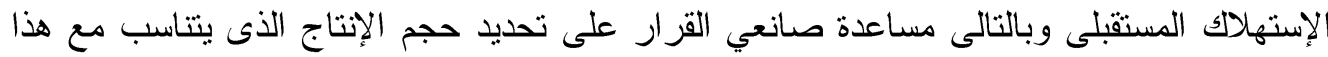

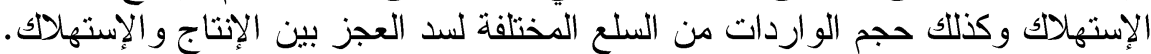

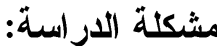

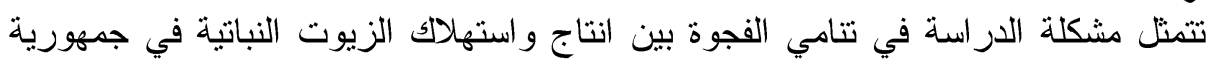

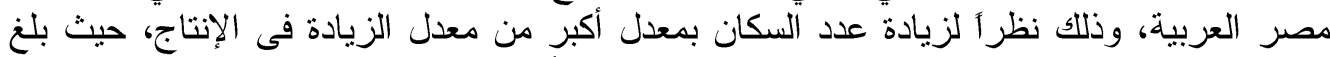

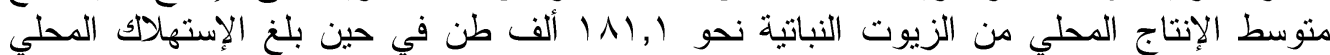

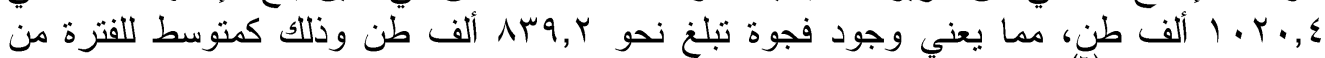

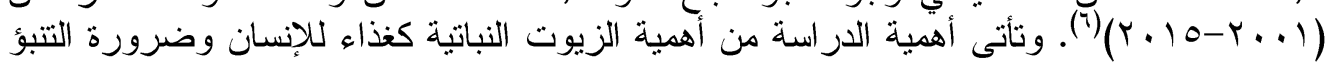

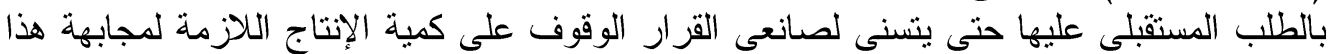

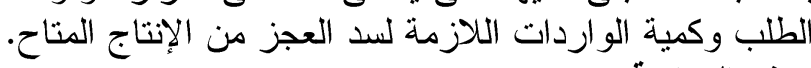

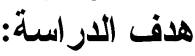

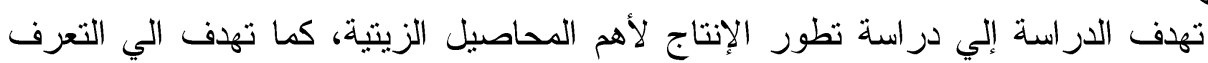

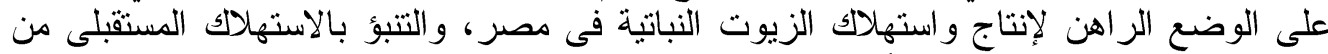

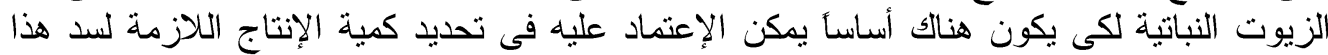

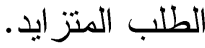

Fayoum J. Agric. Res. \& Dev., Vol. 32, No.2, July, 2018 


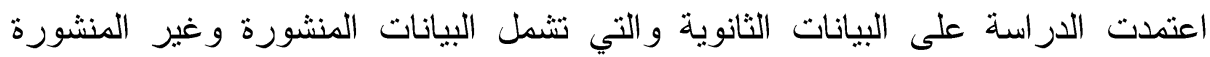

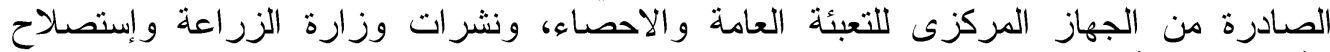

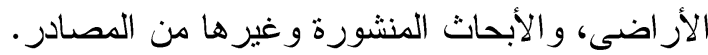

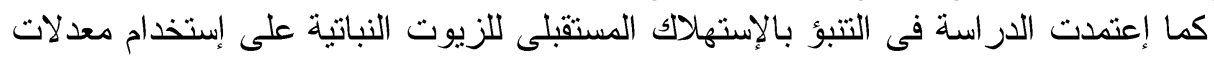

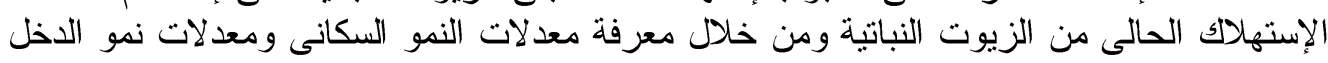

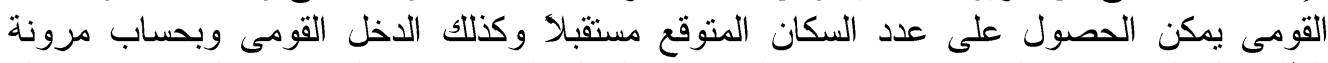

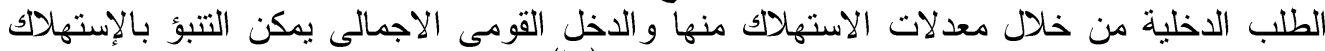

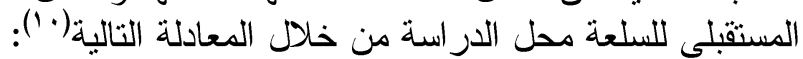

$\log \left(y_{1}\right)=a+b * \log \left(x_{1}\right)$

$\log \left(y_{0}\right)=a+b^{*} \log \left(x_{0}\right)$

الاستهلاك الدخل الأل income; y = consumption

$\log \left(y_{1} / y_{0}\right)=b * \log \left(x_{1} / x_{0}\right)$

$\mathrm{y}_{1} / \mathrm{y}_{0}=\operatorname{EXP}\left(\mathrm{b} * \operatorname{LN}\left(\mathrm{x}_{1} / \mathrm{x}_{0}\right)\right)$

$\mathrm{y}_{1}=\mathrm{y}_{0} * \operatorname{EXP}\left(\mathrm{b} * \operatorname{LN}\left(\mathrm{x}_{1} / \mathrm{x}_{0}\right)\right)$

\section{Where:}

$\mathrm{y}_{0}=$ Base year per capita consumption

متوسط نصيب الفرد كجم/ سنة لسنة الاساس

$\mathrm{y}_{1}=$ Horizon year per capita consumption

منتوسط نصيب الفرد كجم/ سنة لسنة التنبؤ

b =x coefficient (income elasticity) مرونة الطلب الدخلية

X0 = Base year per capita expenditure (GDP)

متوسط نصيب الفرد من الاخل القومى فى سنة الاساس

X1 = Horizon year per capita expenditure (GDP)

متوسط نصيب الفرد من الدخل القومى فى سنة التنبؤ

X1/X0 = Index of per capita GDP

أنتائج ومناقشتها:

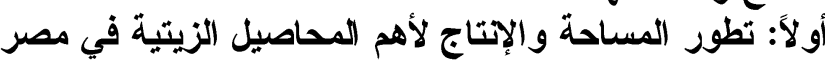

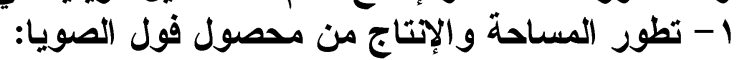

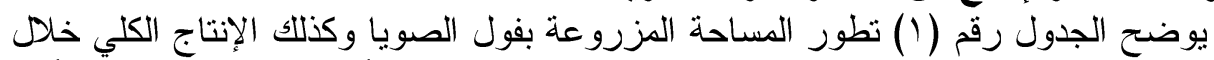

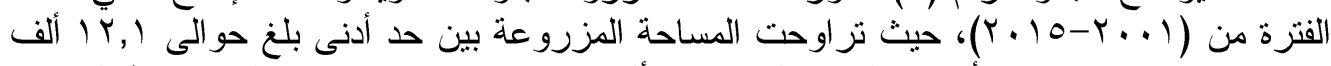

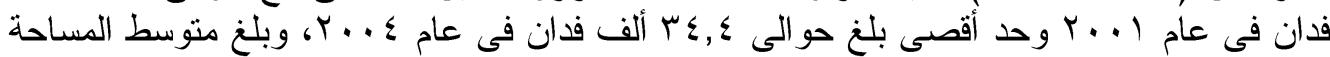

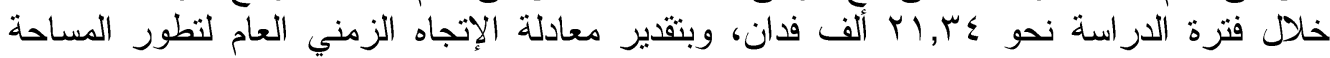

Fayoum J. Agric. Res. \& Dev., Vol. 32, No.2, July, 2018 
$\wedge \wedge$

يتضح من المعادلة رقم(1) بالجدول رقم (r) أنها أخذت اتجاها عاما متزايدا ومعنوي احصائياً

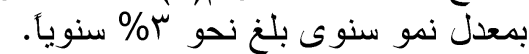

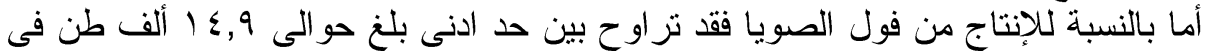

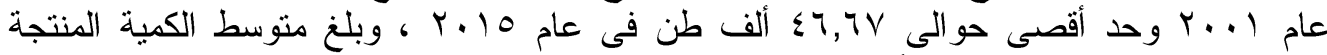

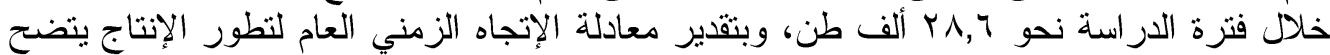

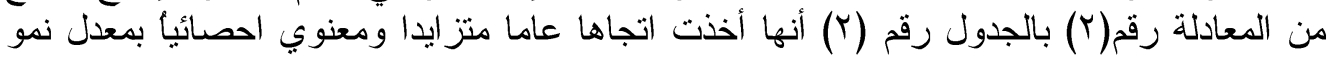

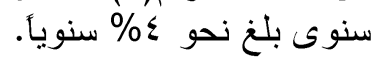

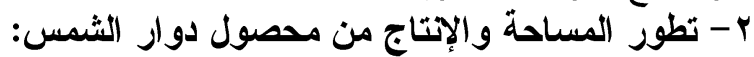

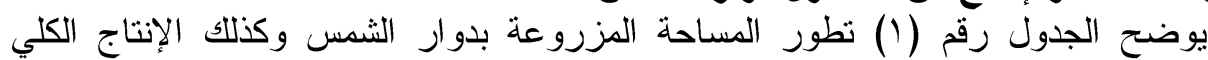

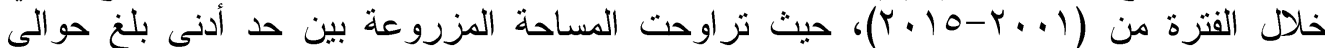

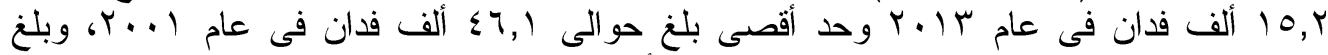

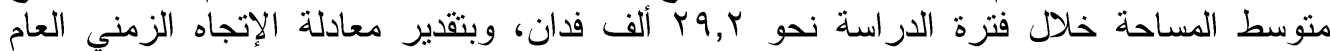

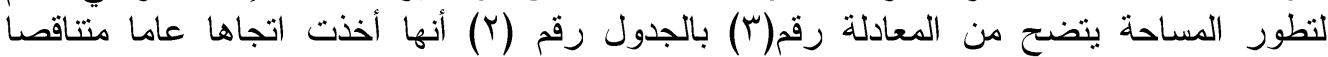

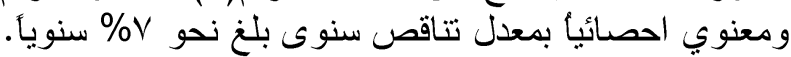

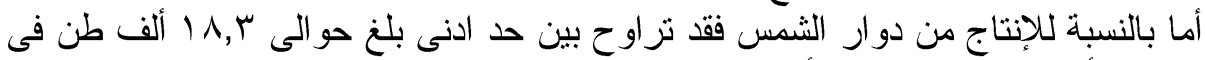

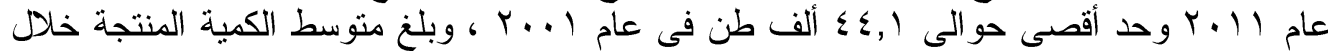

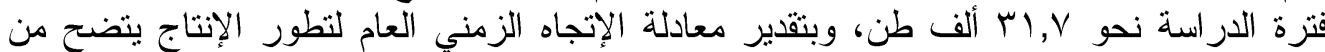

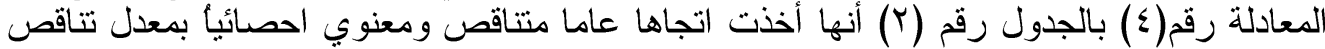

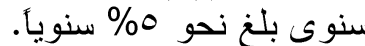
ب- تطور المساحة والإتتاج من محصول الفول السوداني:

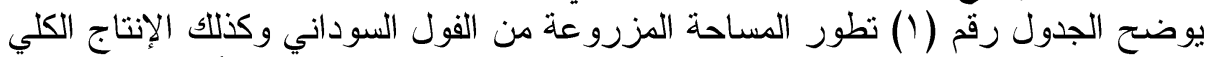

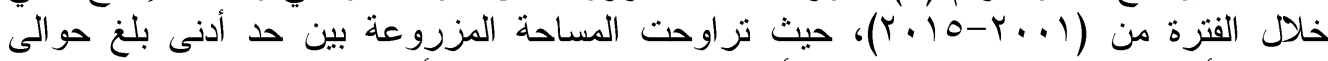

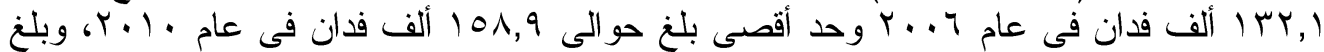

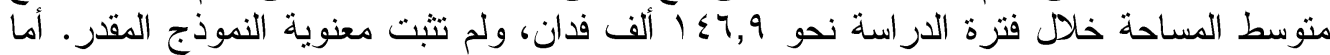

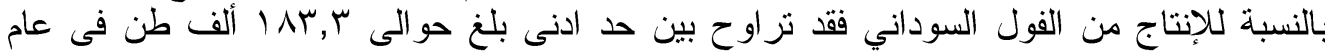

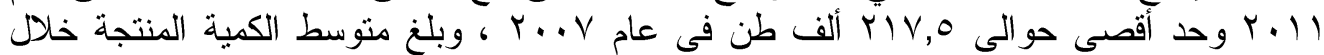

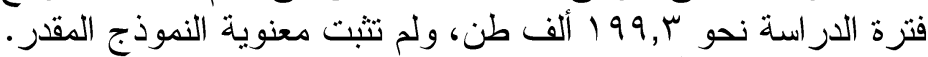

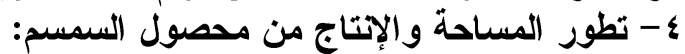

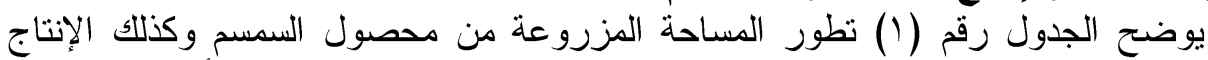

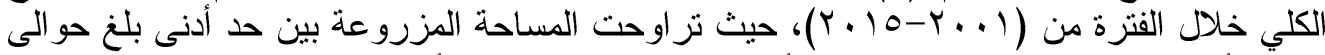

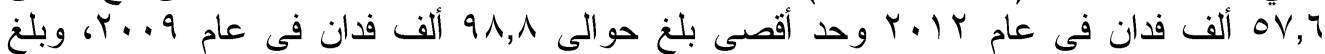

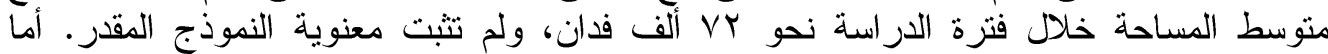

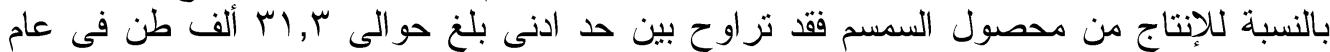

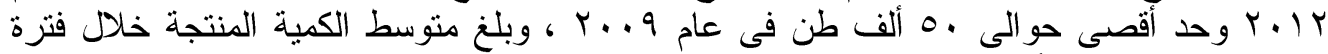

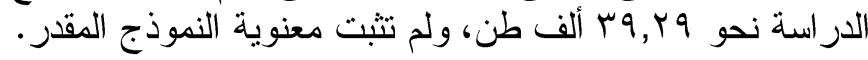

Fayoum J. Agric. Res. \& Dev., Vol. 32, No.2, July, 2018 
$\Lambda 9$

جدول (1): تطور المساحة والإتتاج لأهم المحاصيل الزيتية في مصر خلا الفترة (1 (.. ب-

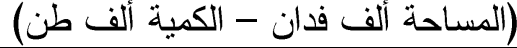

\begin{tabular}{|c|c|c|c|c|c|c|c|c|}
\hline \multicolumn{2}{|c|}{ السمسم } & \multicolumn{2}{|c|}{ الفول السوداني } & \multicolumn{2}{|c|}{ دوار الشمس } & \multicolumn{2}{|c|}{ فول الصويا } & \multirow{2}{*}{ سنة المحصول } \\
\hline الإلتاج & المساحة & الإنتاج & المساحة & الإنتاج & المساحة & الإنتاج & المساحة & \\
\hline$r \varepsilon . \wedge$ & 78.9 & $r .0 .1$ & $10 . .1$ & $\varepsilon \varepsilon .1$ & $\varepsilon 7.1$ & $1 \varepsilon .9$ & IY.V & $r+1$ \\
\hline M. & VY.I & 191 & $1 \leqslant 1.1$ & ro & H.r.r & $1 \mathrm{~V} . \mathrm{V}$ & $1 \varepsilon .1$ & $r \ldots r$ \\
\hline r.V & VI.0 & 190.9 & $I \leqslant V . Y$ & M. & TY.E & r^.V & 19.8 & $r \ldots r$ \\
\hline 15.9 & 79.7 & 191.2 & $1 \leqslant \varepsilon$ & $\varepsilon \psi . \varepsilon$ & $\leqslant 0.0$ & $\varepsilon r . \varepsilon$ & $r \varepsilon .1$ & $r \ldots \varepsilon$ \\
\hline M.V & 97.9 & $199 . \%$ & $1 \leqslant \Lambda$ & $r \cdot . \varepsilon$ & W1.0 & $r \bullet . \Lambda$ & $r . .1$ & $r \ldots o$ \\
\hline$\varepsilon+.7$ & VT.\& & 1Ar.9 & ITY.I & $r 0 . \Lambda$ & $r 0.7$ & $r r$ & $1 V . \Lambda$ & $Y .+Y$ \\
\hline$\$ 1.0$ & $V \varepsilon .9$ & YIV.0 & $100 . r$ & TV. & YV.r & ro. & 11.0 & $r+v$ \\
\hline M.० & 77.8 & $\uparrow . \wedge . \wedge$ & $1 \leqslant 7 . Y$ & rq. & Yq.r & Yq.Y & $Y . . V$ & $Y . \Lambda$ \\
\hline 0 & $9 \wedge . \wedge$ & 191 & 101.9 & ro. & r9.7 & YY.E & $r . . V$ & $r . . q$ \\
\hline \&7.Y & $V \varepsilon .9$ & $Y, Y$ & 101.9 & $M . . \Lambda$ & ro.r & $r r . r$ & 18.1 & $Y .1$. \\
\hline$\varepsilon r . Y$ & $\vee \wedge . r$ & $r .7 .0$ & $10 \leqslant .1$ & $1 \wedge . r$ & $1 V .0$ & rq.1 & YY.V & $r .11$ \\
\hline$r . r$ & 0 V. 7 & $Y .0 . \varepsilon$ & $1 \leqslant \Lambda . V$ & $Y$. & IV.V & Y0.9 & 18.1 & $Y .1 Y$ \\
\hline TY.V & 09.71 & $r . \varepsilon . \Lambda$ & $1 \leqslant V . \Lambda$ & 19 & 10.1 & TY.VE & YY.E & $r .1 T$ \\
\hline rV.\&V & Ir.Ar & IAT.r & $1+\varepsilon . \varepsilon$ & YI.Or & IT.KY & rq.Ao & Y^.० & $Y .1 \varepsilon$ \\
\hline$\{\Lambda .11$ & $\Lambda \leq . \Psi_{1}$ & $19 \mathrm{V.r}$ & $1 \leq r$ & YY.Y & $10 . V$ & $\varepsilon 7.7 \mathrm{~V}$ & rr.9 & 1.10 \\
\hline rq.rq & VY & $199 . \%$ & $1 \leqslant 7.9$ & rI.V & rq.r & YА. & YI.Y\& & المتوسط \\
\hline
\end{tabular}

المصدر: وزارة الزراعة واستصلاح الأراضي، قطاع الثئون الاقتصادية، الإدارة المركزية للاقتصاد الزراعي، أعداد

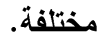

جدول (r): معادلات الإتجاه الزمني العام لكل من المساحة والإنتاج لأهم المحاصيل الزيتية في

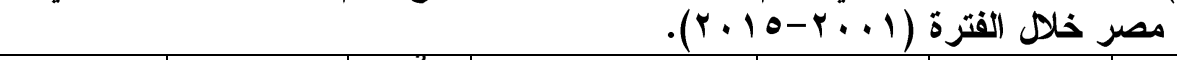

\begin{tabular}{|c|c|c|c|c|c|c|}
\hline السنوي النمو & $F$ & $\mathbf{R}^{2}$ & المعادلة & رقم المعادلة & المتغير & البيان \\
\hline$r$ & $*(\bullet . r)$ & $\cdots \leqslant \wedge$ & 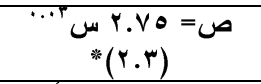 & 1 & المساحة & \multirow{2}{*}{ فول الصويا } \\
\hline$\varepsilon$ & $* *(\mathrm{~V} .0)$ & 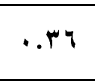 & $\begin{array}{c}\cdots \xi \varepsilon r . q V=ص \\
* * *(r . V)\end{array}$ & $r$ & الاتتاج & \\
\hline$v-$ & $* *(\Gamma \bar{r})$ & $\because v$ & 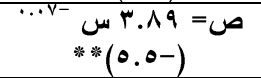 & $r$ & المساحة & \multirow{2}{*}{ دوار الثمس } \\
\hline 0- & $* *(Y) . V)$ & $.7 r$ & 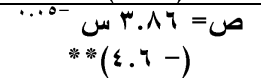 & $\varepsilon$ & الانتاج & \\
\hline$\ldots+1-$ & $(\cdots \cdots 1)$ & $\cdots+1$ & 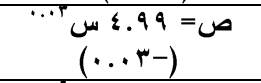 & 1 & المساحة & \multirow{2}{*}{ لفول السوداني } \\
\hline$\cdot, \ldots$ & $(\cdots 0)$ & $\ldots r$ & 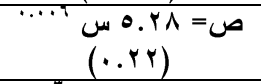 & $r$ & الانتاج & \\
\hline$\ldots r-$ & $(\cdots+1)$ & $\ldots 1$ & 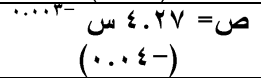 & $r$ & المساحة & \multirow{2}{*}{ السمسم } \\
\hline$\ldots v$ & $(\cdot . \wedge)$ & $\ldots 0$ & 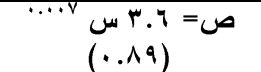 & $\varepsilon$ & الاتتاج & \\
\hline
\end{tabular}

( ) القيم الموجودة بين الاقواس أسفل معاملات الاتحدار تثير الي قيم (t) المحسوبة.

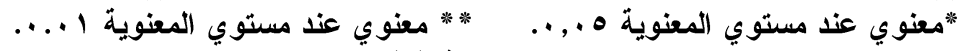

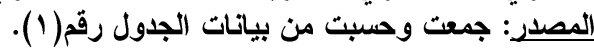

Fayoum J. Agric. Res. \& Dev., Vol. 32, No.2, July, 2018 
9.

ثانياً: الوضع الر اهن لإنتاج واستهلاك الزيوت الغذائية في مصر

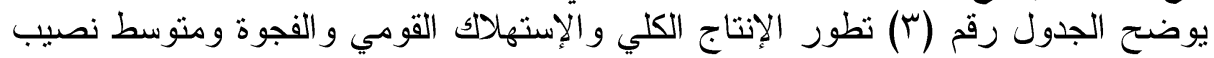

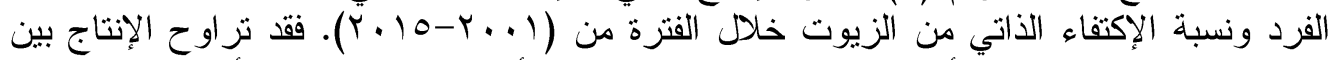

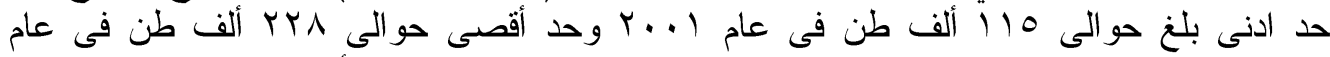

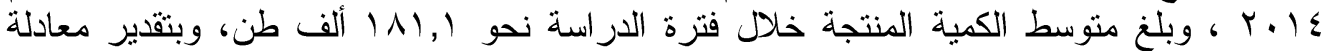

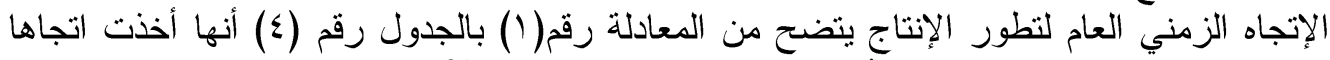

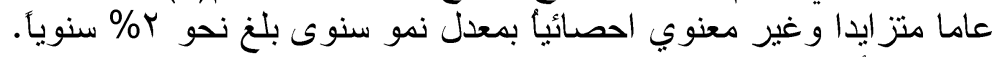

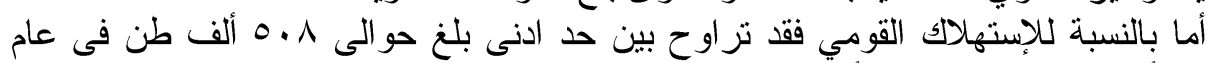

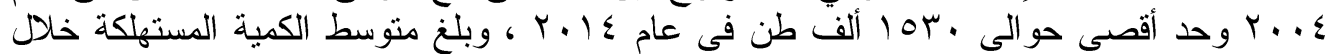

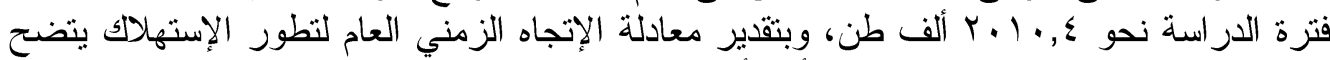

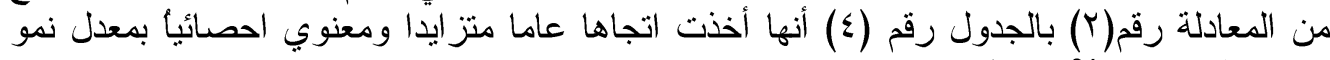

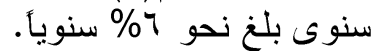

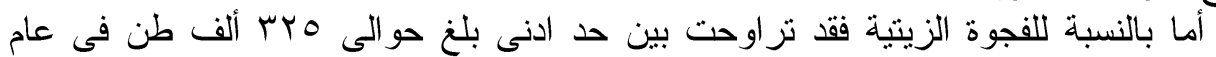

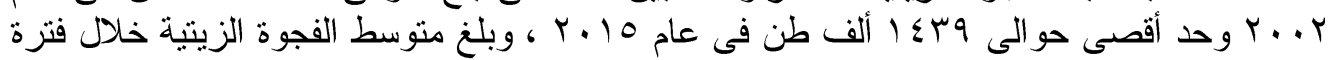

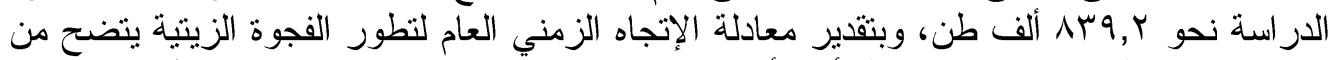

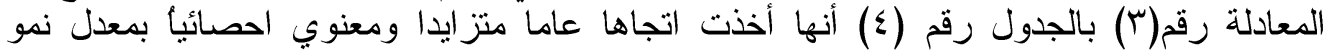

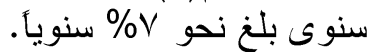

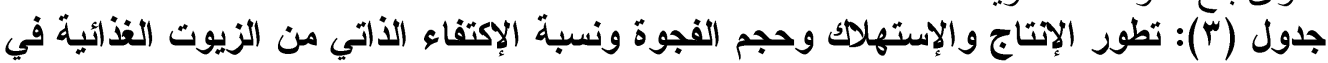

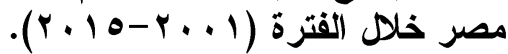

\begin{tabular}{|c|c|c|c|c|c|}
\hline نسبة الإكتفاء الذاتي & متوسط نصيب الفرد & ألفجوة الزيتية & الإستهلهاك & ألف طإتناج & لسنة البيان \\
\hline$r, 1$ & 11.7 & $\varepsilon O r$ & $V \leqslant \Lambda$ & 110 & $r \ldots 1$ \\
\hline$r \varepsilon, Y I$ & V.r & rro & $\Delta V \varepsilon$ & $|r|$ & $r \ldots r$ \\
\hline r४,०V & V.o & $r V \mu$ & \&9 & 179 & $r \ldots r$ \\
\hline 10,1 & $1 \leqslant .0$ & No. & 0.1 & $1 \% 0$ & $r \ldots \varepsilon$ \\
\hline $17, r \leqslant$ & IV.V & $1, \varepsilon \leqslant$ & $1 \ldots 1$ & 101 & $r \ldots o$ \\
\hline $1 \leqslant, 9$. & $19 . \%$ & IIAr & $T r \leqslant \Lambda$ & Y.乏 & $r \ldots q$ \\
\hline$r 1,00$ & $1 . . v$ & OrA & $1+199$ & $r \cdot V$ & $r \ldots v$ \\
\hline$\overline{Y \mu, V V}$ & 9.1 & 071 & VA५ & $r \leqslant \Lambda$ & $r \ldots 1$ \\
\hline$r \varepsilon, 10$ & $9 . \varepsilon$ & OrA & VWY & IVo & r..q \\
\hline$r 0, q 4$ & $\Lambda .0$ & $\varepsilon q v$ & $V I \varepsilon$ & $1 \times 4$ & r.1. \\
\hline $10, v_{0}$ & $10 . \mathrm{V}$ & $1 \cdot V$. & $7 V 1$ & $1 V \varepsilon$ & $r .11$ \\
\hline IY,V & $1 \varepsilon .7$ & IYAY & ITV. & r... & $r+1 r$ \\
\hline IY,q. & 11.1 & $T H \cdot T$ & $1 \leqslant 01$ & $17 V$ & $r \cdot 1 T$ \\
\hline $10, .4$ & 10.5 & $11 \% 0$ & 104. & YYA & $r+1 \leqslant$ \\
\hline $1 \cdot, \mathrm{rT}$ & $v .4$ & $1 \leqslant r q$ & ITrE & 199 & $r .10$ \\
\hline$r \cdot, . q$ & TY.r & Arq.Y & $1 \cdot r \cdot . \varepsilon$ & $1 \mathrm{A1.1}$ & المتوسط \\
\hline
\end{tabular}

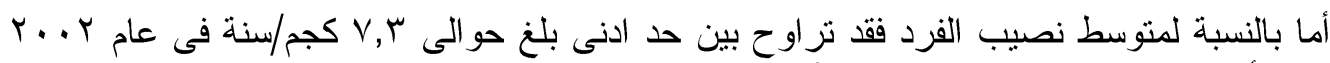

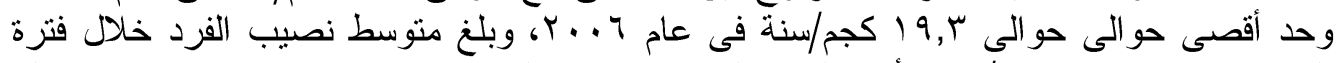

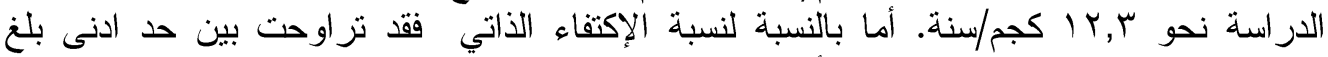

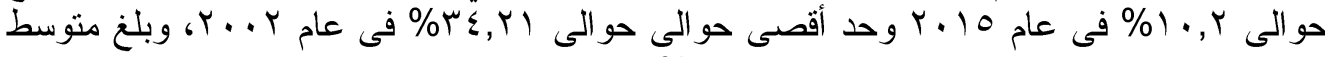

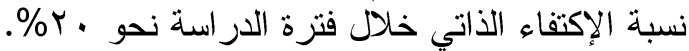

Fayoum J. Agric. Res. \& Dev., Vol. 32, No.2, July, 2018 
91

جدول (ع): معادلات الإتجاه الزمني العام لكل من الإنتاج والإستهلاك وحجم الفجوة الزيتية في

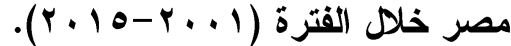

\begin{tabular}{|c|c|c|c|c|c|}
\hline معدل النمو السنوي\% & $\mathbf{F}$ & $\mathbf{R}^{2}$ & المعادلة & رقم المعادلة & المتغير \\
\hline$r$ & $(\Gamma . \varepsilon)$ &.$r \varepsilon$ & 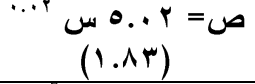 & 1 & الانتاج \\
\hline 7 & $* *(I r)$ & $\cdots 0$ & 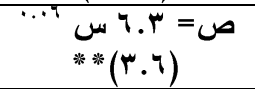 & $r$ & الإستهلاك \\
\hline V & $* *(11 . \wedge)$ & $\because \leqslant V$ & 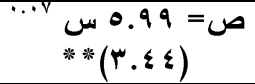 & $r$ & الزجوة الزيتية \\
\hline
\end{tabular}

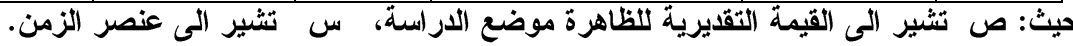

( ) القيم الموجودة بين الاقو اس أسفل معاملات الاتحدار تثثير الي قيم (t) المحسوبة.

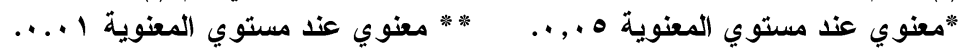

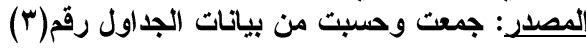

ثالثاً: أثر الاخل القومى على الطلب من الزيوت النباتية في مصر:

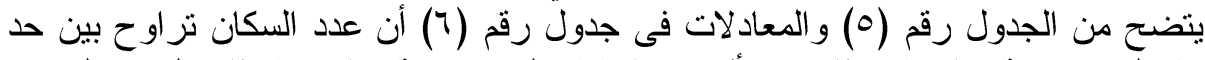

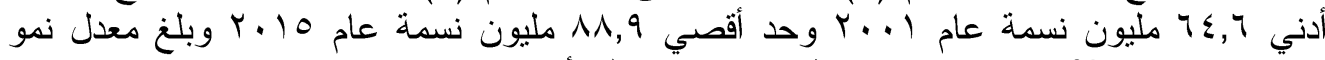

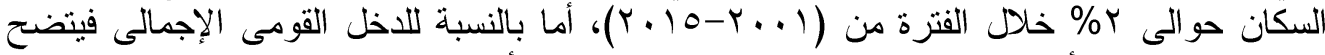

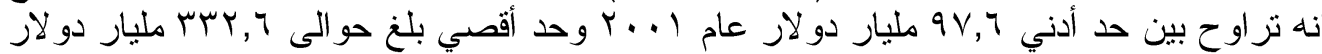

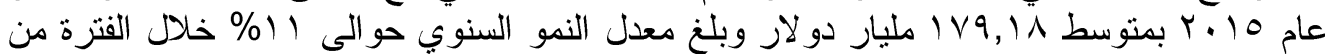

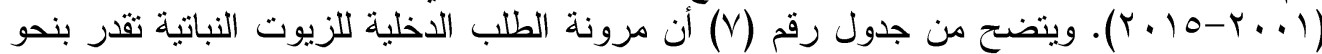

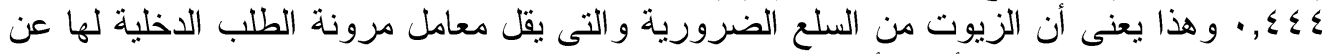

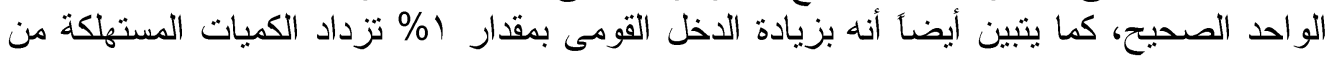

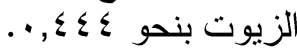

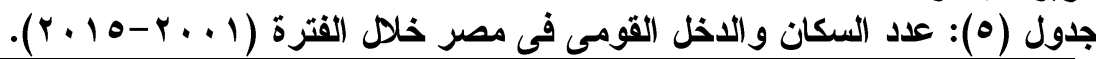

\begin{tabular}{|c|c|c|}
\hline الاخل القومى بالمليون دولار & عدد السكان (ألف نسمة) & السنة \\
\hline 9V7TY & T\&YOY & $r \ldots 1$ \\
\hline$\wedge \vee \wedge 0, . \vee$ & 70919 & $r \ldots r$ \\
\hline AYqY & TVMIT & $r \ldots r$ \\
\hline$\nabla \wedge \wedge \leq 0 . Y$ & 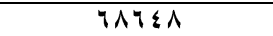 & $r \ldots \varepsilon$ \\
\hline 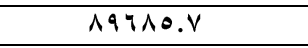 & $7999 V$ & $r \ldots o$ \\
\hline $1 \cdot V \leqslant \Lambda \varepsilon$ & VIr $\leqslant \wedge$ & $r \ldots q$ \\
\hline $17 \cdot \leqslant V q$ & VYq\&. & $r \ldots v$ \\
\hline IтrAIA.r & $V \leqslant \varepsilon r q$ & $r \ldots \lambda$ \\
\hline 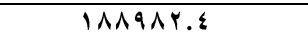 & 87.99 & $r \ldots q$ \\
\hline 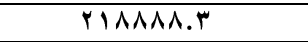 & $\vee \vee \wedge \varepsilon$. & $r .1$. \\
\hline r r...1.9 & V9411 & $r .11$ \\
\hline rVARVY.A & 1107V & $r+1 r$ \\
\hline 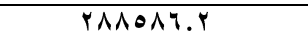 & ATYTV & $r .1 r$ \\
\hline r.00rq.V & 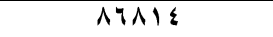 & $r+1 \varepsilon$ \\
\hline PrYYqA & $\wedge \wedge ৭ \diamond \wedge$ & $r .10$ \\
\hline IVqIA0.Y & VOrYO.V & المتوسط \\
\hline
\end{tabular}

المصدر: أ الجهاز المركزي للتعبئة العامة والاحصاء، الكتاب الإحصائى السنوى - السكان، اعداد متفرقة.

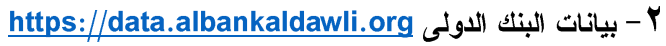
** تم استخدام الدولار لتفادي مشكلة التضخم

Fayoum J. Agric. Res. \& Dev., Vol. 32, No.2, July, 2018 
qr

\begin{tabular}{|c|c|c|c|c|c|}
\hline معدل النمو & $\dot{\theta}$ & ru & $ت$ & معادلة الاتجاه الزمنى العام & البيان \\
\hline$\%$ \% & *** $(Y+11)$ &., 99 & $* *(\xi \Lambda)$ & ص^ = = 11س & عدد السكان \\
\hline$\% 11$ & **** $(170)$ & $\cdot, 94$ & $* *(\mid r . \wedge)$ & 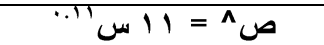 & الدخل القومى بالمليون دولار \\
\hline
\end{tabular}

المصدر: جمعت وحسبت من بياتات جدول رقم (ه) مغنوية

جدول (V): معادلات الاتجاه الزمنى العام لأثز الاخل القومى على الطلب من الزيوت النباتية خلال

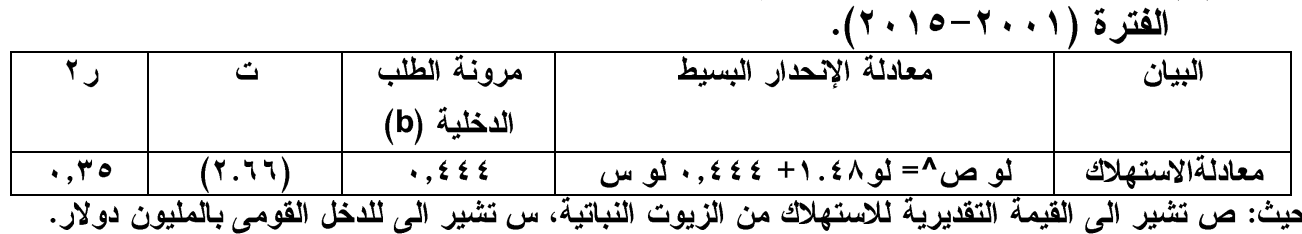

المصدر: جمت وحسبت من بياتات الجداول رقم (ب)، (ه).

رابعاً: التتبؤ المستقبلى بإستهلالك الزيوت النباتية في مصر : الإستو

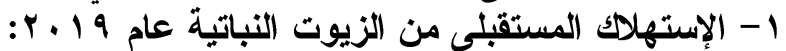

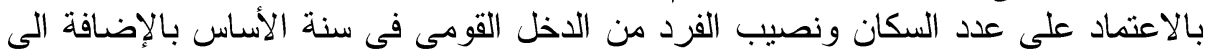

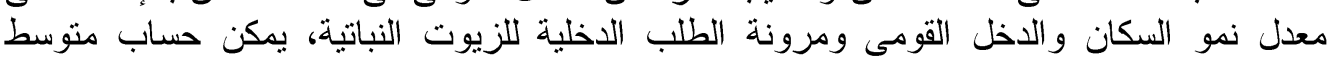

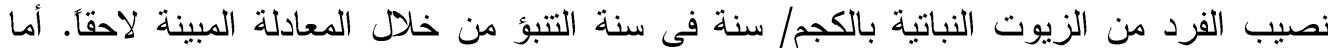

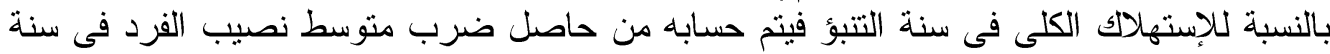
التتبؤ مضروبأ فى عدد السكان المنوقع فى سنة التنبؤ .

$\mathrm{y}_{1}=\mathrm{y}_{0} * \operatorname{EXP}\left(\mathrm{b} * \operatorname{LN}\left(\mathrm{x}_{1} / \mathrm{x}_{0}\right)\right)$

$\mathrm{y} 1=11.6 * \operatorname{EXP}(0.444 * \operatorname{LN}(7529.1 / 1510.12))$

$\mathrm{y} 1=23.61$

الاستهلاك على المستوى القومى فى سنة التتبؤ = متوسط نصيب الفرد (y1 ) * عدد السكان المتوقع

Fayoum J. Agric. Res. \& Dev., Vol. 32, No.2, July, 2018 
94

جدول(^): عدد السكان والاخل القومى ومتوسط نصيب الفرد من الاخل القومى فى سنة الأساس

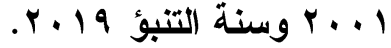

\begin{tabular}{|c|c|}
\hline القيمة & البيان \\
\hline$T \leqslant Y 0 Y$ & عدد السكان في سنة الاساس | ... بالألف \\
\hline qVYTr & الدخل القومى بالمليون دو لار في سنة الاساس | ... \\
\hline $101 \cdot .14$ & نصيب الفرد من الدخل القومى بالدو لار في سنة الاساس | +. T \\
\hline$r$ & معدل نمو السكان \% \\
\hline 11 & معدل نمو الاخل القومى \% \\
\hline $9 \leqslant 1 \wedge 7$ & عدد السكان المتوقع في سنة التتبؤ 9 (ب بالألف \\
\hline$V .91 \% 0$ & الدخل القومى المتوقع بالمليون دو لار في سنة التنبؤ 19 . \\
\hline vorq.1 & نصيب الفرد من الدخل القومى بالدو لار في سنة التتبؤ 9 ب. \\
\hline$V \leqslant \Lambda$ & الاستهلاك الكلى من الزيوت في سنة الاساس I . . ب بالألف طن \\
\hline 11.7 & متو سط نصيب الفرد من الزيوت في سنة الاساس كجم/سنة \\
\hline.$+ \leqslant \varepsilon \varepsilon$ & مرونة الطلب الدخلية \\
\hline rr.YI & متوسط نصيب الفرد من الزيوت في سنة التتبؤ 9 ـ ب كجم/سنة \\
\hline TrYE & الاستهلاك الكلى من الزيوت في سنة التتبؤ 9 أ ب بالألف طن \\
\hline
\end{tabular}

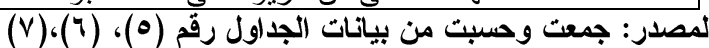

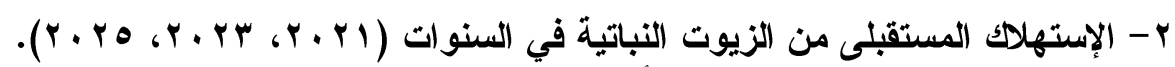

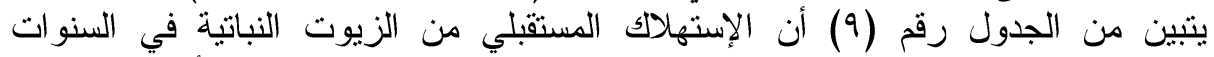

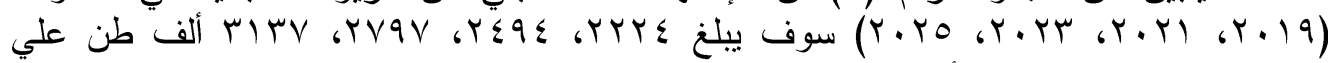

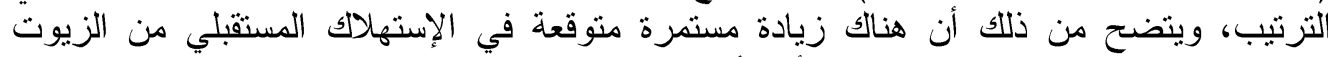

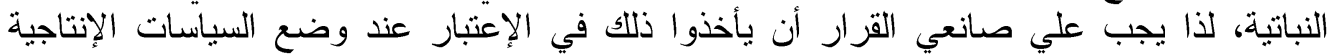

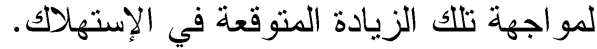

\begin{tabular}{|c|c|c|c|c|}
\hline \multirow{2}{*}{ r. ro } & \multirow{2}{*}{$r+r r$} & \multirow{2}{*}{$r \cdot r+$} & \multirow{2}{*}{$r .19$} & السنوات \\
\hline & & & & لبيان \\
\hline TITV & rVqV & $T \leq 9 \varepsilon$ & TYYE & الكمية المستهلكة (بالألف طن) \\
\hline
\end{tabular}

المصدر: جمعت وحسبت من جداول رقم (؟)، (V)، (^).

الملخص و التوصيات:

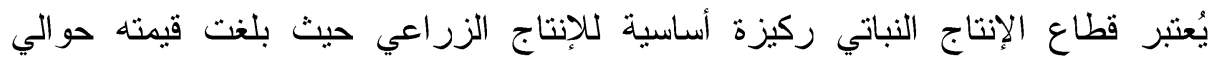

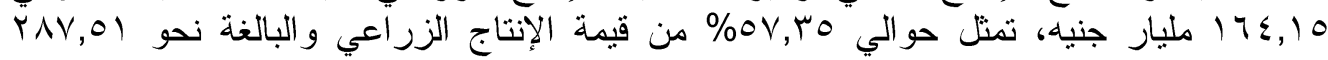

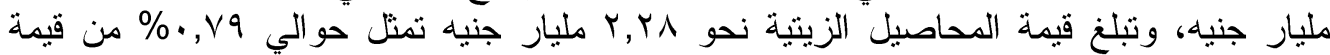

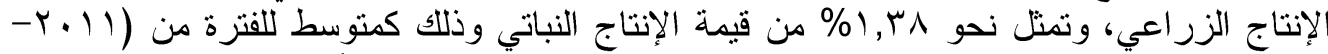

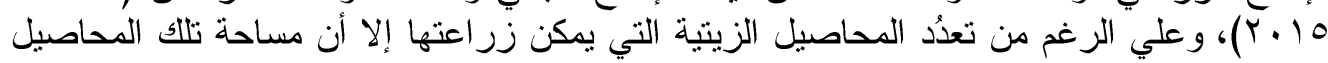

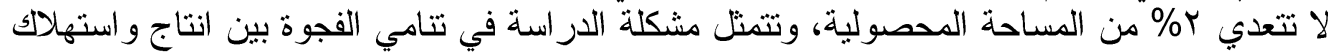

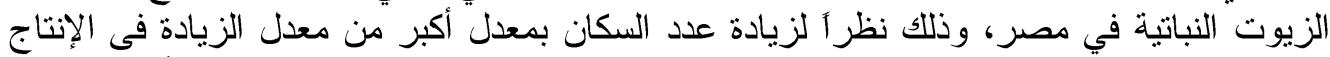

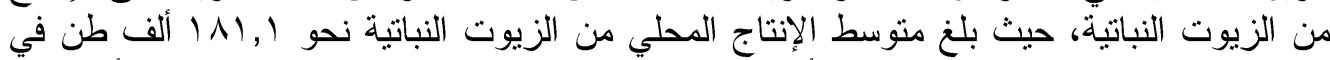

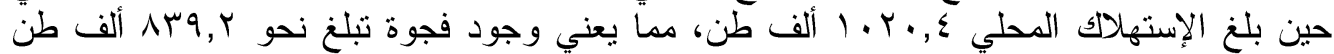

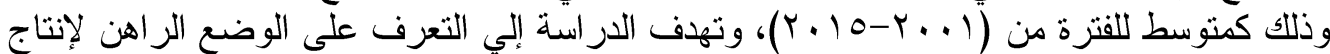

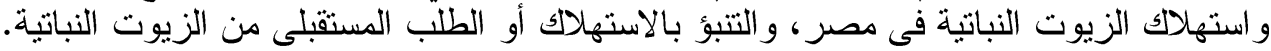

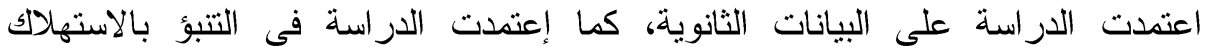

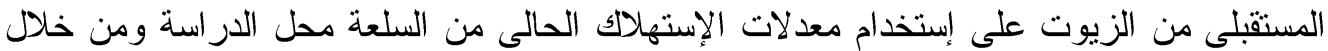

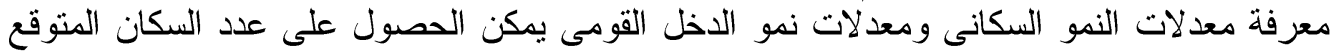

Fayoum J. Agric. Res. \& Dev., Vol. 32, No.2, July, 2018 
$9 \varepsilon$

مستقبلا وكذلك الاخل القومى وبحساب مرونة الطلب الدخلية للسلعة من خلال معدلات الاستهلاك

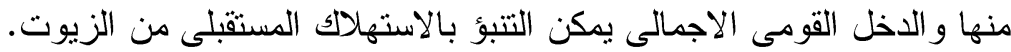

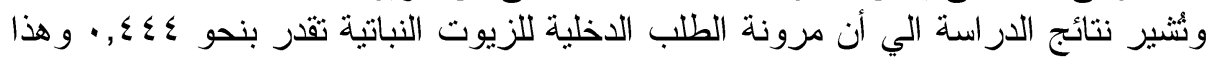
يعنى أن الزيوت من السلع الضرورية والتى يقل معامل مرونة الطلب الاخلية لها عن الونة الواحد

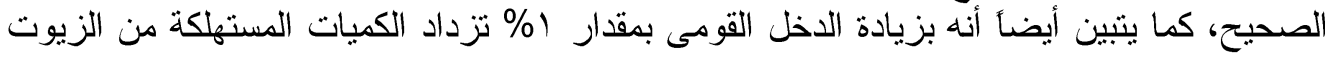

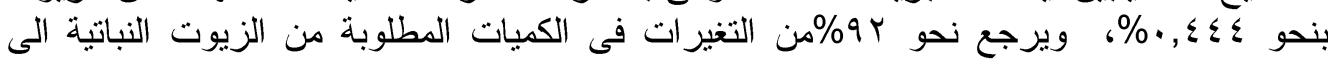

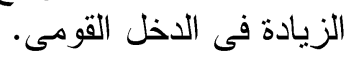

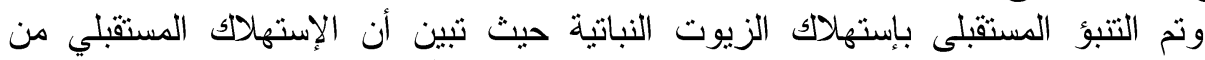

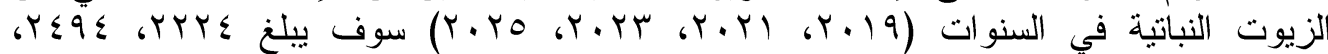
الإنه

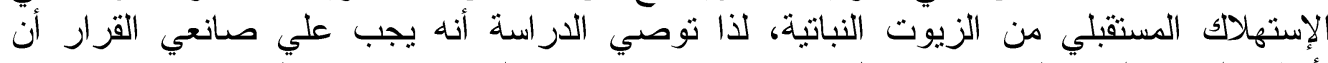
يأخذوا الزيادة المتوقعة في الإستهلاك في الإعتبار عند وضع السياتيات الإنيات الإنتاجية.

المر اجع:

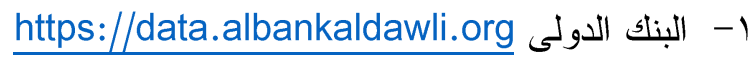

ץ- الجهاز المركزي للتعبئة العامة والاحصاء،الكتاب الإحصائى السنوى -السكان، أعداد مختلفة.

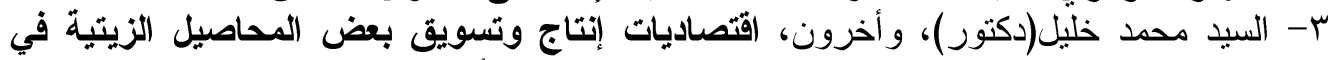

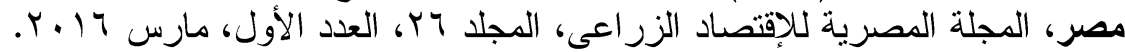

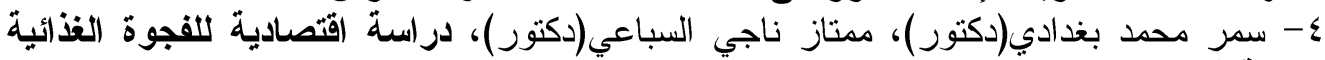

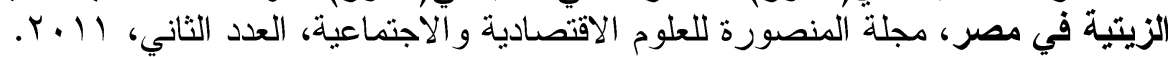

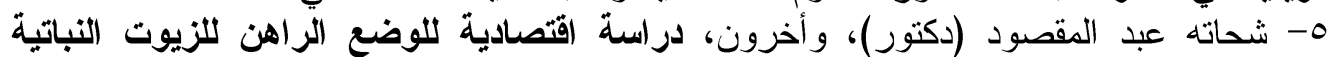

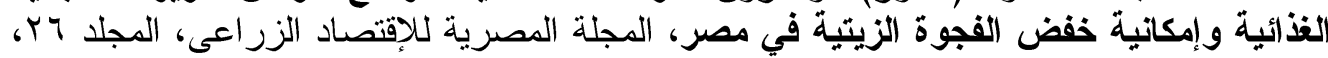

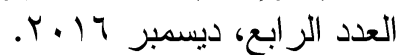
4- هناء شداد عبد اللطيف دئدكتور)، الإتجاهات الإتتاجية والإستهلاكية للزيوت النباتية في مصر،

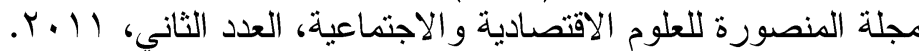

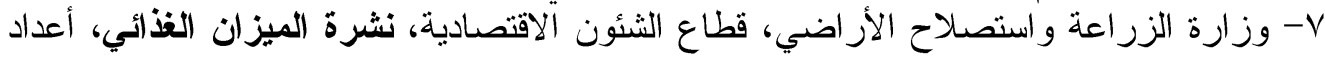

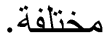

^- وزارة الزراعة و استصلاح الأراضي، قطاع الثئون الاقتصادية، الإدارة المركزية للاقتصاد الزر اعي، أعداد مختلفة.

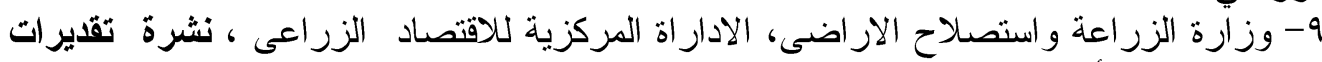

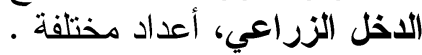

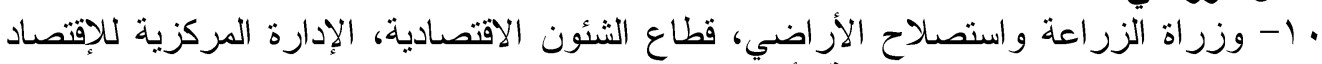

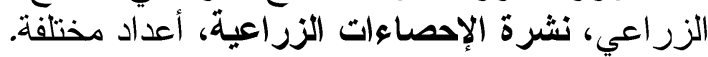
11-Abdel Aziz Ibrahim,Policy Analysis Exercise: Food Demand Projection, Food Security Policy and Strategy Capacity Building Programme (FSPS). 12- Allen, R.G.A, Mathematical Analysis for Economists, Macmillan St, Martin Press London, 1972.

Fayoum J. Agric. Res. \& Dev., Vol. 32, No.2, July, 2018 


\title{
ECONOMIC STUDY OF EDIBLE OILS AND FUTURE PREDICTION OF DEMAND IN EGYPT
}

Dr/Mahmoud Abdelsalam Ahmed*

*Department of Agricultural Economics, Faculty of Agriculture, Fayoum

University.

\begin{abstract}
:
The plant production sector is amajor pillar of agricultural production, with a value of about 164.15 billion pounds, representing about $57.35 \%$ of the value of agricultural production amounting to about 287.51 billion pounds. the value of oil crops is about 2.28 billion pounds, representing about $79 \%$ of the value of agricultural production, representing about $1.38 \%$ of the value of plant production.

In spite of the multiplicity of oil crops that can be cultivated, the area of these crops does not exceed $2 \%$ of the crop area. the problem of the study is the growing gap between the production and consumption of edible oils in Egypt, due to the increase in the population rate higher than the rate of increase in production of edibl oils.

Where the average domestic production of edible oils amounted to 181.1 thousand tons while the local consumption amounted to 1020.4 thousand tons, which means a gap of about 839,2 thousand tons as an average for the period (2001-2015), the study aims to identify on the current status of production and consumption of edible oils in Egypt, and forecast consumption of edible oils .

The study relied on secondary data, it is also relied on the prediction of the future consumption of edible oils by using the current consumption rates of oils, and by knowing the population growth rates and the rates of growth of the national income.

The results of the study indicate that the elasticity of the income demand for edible oils is estimated at 0.444 . This means that edible oils is essential commodity, also shows that by increasing the national income by $1 \%$, the quantities consumed of oils increasing by $0.444 \%$.

Future consumption of edible oils was predicted in the future (2019, 2021, 2023 and 2025) which is 2224, 2494, 2797 and 3137 thousand tons respectively. it is clear that there is an expected continuous increase in the future consumption of edible oils, so decision-makers should take this into consideration when putting productivity policies to cope with the expected increase in consumption.
\end{abstract}

Fayoum J. Agric. Res. \& Dev., Vol. 32, No.2, July, 2018 